\title{
DETERMINAÇÃO DE DESIGNER DRUGS EM SALIVA POR PAPER SPRAY MASS SPECTROMETRY
}

\author{
Lanaia Ítala L. Maciela, Thays C. Carvalho ${ }^{a}$, Igor Pereira ${ }^{a}$ e Boniek G. Vaz*,a, \\ Instituto de Química, Universidade Federal de Goiás, 74690-900 Goiânia - GO, Brasil
}

Recebido em 23/01/2019; aceito em 06/05/2019; publicado na web em 27/06/2019

\begin{abstract}
DETERMINATION OF DESIGNER DRUGS IN SALIVA BY PAPER SPRAY MASS SPECTROMETRY. Determination of two drugs of abuse in saliva has been performed by Paper Spray Ionization Mass Spectrometry (PSI-MS) using a Molecularly Imprinted Polymer (MIP) coated paper as substrate. The method has been optimized for quantification of 3,4-methylenedioxy$N$-methylamphetamine (MDMA) and methamphetamine, 3,4-methylenedioxyamphetamine (MDA) from synthetic saliva samples. Rapid analysis ( $1 \mathrm{~min}$ ) of designer drugs in saliva is demonstrated using MIP-PSI-MS. Both precursor ions of MDMA and MDA were confirmed by tandem mass spectrometry (MS/MS) experiments by elucidating the fragmentation patterns formed via collisioninduced dissociation (CID). The MIP-PSI-MS method showed greater selectivity for MDMA and MDA in comparison with other analytes. Signal intensity ratios of the most important transitions for MDMA $(194 \rightarrow 163)$ and MDA $(180 \rightarrow 163)$ were found linear $(\mathrm{R}>0,99)$ in the range 25-500 $\mu \mathrm{g} \mathrm{L} \mathrm{L}^{-1}$. The LOD and LOQ for MDMA were $1.11 \mu \mathrm{g} \mathrm{L}-1$ and $18.73 \mu \mathrm{g} \mathrm{L}^{-1}$, respectively, and for MDA were $3.42 \mu \mathrm{g} \mathrm{L}^{-1}$ and $21.66 \mu \mathrm{g} \mathrm{L}^{-1}$, respectively. The precision and accuracy values were determined below $4 \%$, and the recoveries about $100 \%$, without pre-concentration/enrichment or separation steps. The MIP-PSI-MS method displays the potential for rapid screening for selective quantitative determination of illicit drugs in saliva samples.
\end{abstract}

Keywords: mass spectrometry (MS); paper spray ionization; molecularly imprinted polymer (MIP); MDMA; MDA.

\section{INTRODUÇÃO}

Os primeiros contatos entre seres humanos e substâncias psicoativas ocorreram através do consumo de plantas. A partir de então, foram isolados alguns metabólitos secundários de origem botânica, como: morfina, cocaína e efedrina. Porém, foi com o aparecimento das anfetaminas que se deu início às primeiras sínteses de substâncias psicoativas inspiradas em produtos naturais. Com os avanços das drogas sintéticas, houve então a popularização das designer drugs. ${ }^{1}$

Designer drugs é o termo utilizado para denominar substâncias sintéticas com efeitos similares aos das drogas ilícitas (substâncias de uso proibido). Essas drogas são produzidas através de ligeiras modificações nas estruturas químicas de substâncias já existentes com o intuito de fraudar a legislação atual em vigor. ${ }^{2}$ As designer drugs têm como característica principal o fato de terem sido modificadas em laboratórios clandestinos, a fim de aumentar, diminuir ou criar efeitos psicoativos. ${ }^{3}$ As mais consumidas são as derivadas dos grupos anfetamínicos (drogas estimulantes), tais como 3,4-metileno-dioxi-anfetamina (MDA) e 3,4-metileno-dioxi-metanfetamina (MDMA). ${ }^{4}$

A correta identificação desta classe de drogas em matrizes biológicas tem sido um grande desafio analítico devido à versatilidade e rapidez pela qual estas moléculas são modificadas. Portanto, faz-se necessário abordagens analíticas diretas e rápidas para a identificação inequívoca das designer drugs. Dentre estas abordagens analíticas, a espectrometria de massas (MS - do inglês, mass spectrometry) vem se destacando por ser uma técnica capaz de detectar, identificar e quantificar moléculas de diversos tamanhos, composições em diferentes matrizes. ${ }^{5}$

Com o surgimento da espectrometria de massas ambiente (AMS), em 2004, principalmente com a introdução das técnicas de ionização Direct Analysis in Real Time (DART) e Desorption Electrospray Ionization (DESI), tem-se, então, um novo, revolucionário e simples modo de gerar íons em espectrometria de massas. ${ }^{7}$ As técnicas de AMS tem como característica comum a geração de íons em condições

*e-mail: boniek@ufg.br ambientes e a ionização do analito que ocorre diretamente do seu "ambiente natural", ou seja, na própria matriz ou com o uso de superfícies auxiliares ${ }^{5,8}$ Assim, a AMS provocou uma nova revolução na espectrometria de massas, tornando-a mais rápida e universalizando seu campo de atuação, principalmente levando-a ao "mundo real". ${ }^{9}$

Dentre os diversos métodos de ionização ambiente de espectrometria de massas, um de maior simplicidade é o Paper Spray Ionization (PSI) ${ }^{10-12} \mathrm{O}$ PSI foi desenvolvido por Liu et al ${ }^{13} \mathrm{~A}$ ionização ocorre adicionando a solução da amostra, previamente acidificada ou basificada, em um pedaço de papel triangular, e com a aplicação de uma elevada diferença de potencial $(\sim 4 \mathrm{kV})$ no papel, tem-se a formação de um spray. Este spray é composto por gotículas contendo íons e o solvente, que por um processo de dessolvatação tem-se a geração de íons em fase gasosa, por um processo similar ao electrospray (ESI), como ilustrado pela Figura 1.

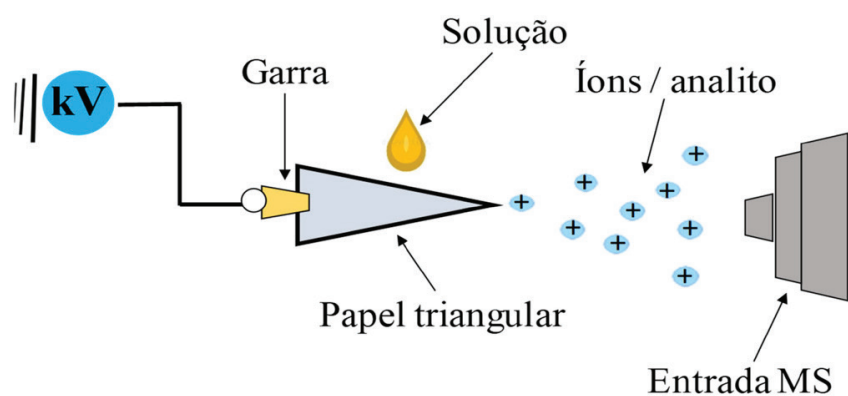

Figura 1. Esquema geral do método de ionização paper spray ionization-PSI

O PSI tem possibilitado a análise de amostras sem o pré-tratamento prévio ou com mínimo preparo de amostra. A sua faixa de aplicação é muito ampla. Inúmeros trabalhos têm demonstrado aplicabilidade do PSI, principalmente em aplicações clínicas, incluindo a triagem neonatal, ${ }^{14}$ monitoramento de drogas terapêuticas, ${ }^{15,16}$ medicina personalizada ${ }^{17,18}$ e a análise de fluidos biológicos, como sangue, ${ }^{19,20}$ urina ${ }^{6,21}$ e saliva. ${ }^{22,23}$ Contudo, toda técnica analítica possui uma limitação inerente, e o PSI apresenta algumas quando aplicado na análise de 


\section{Monômero Funcional}

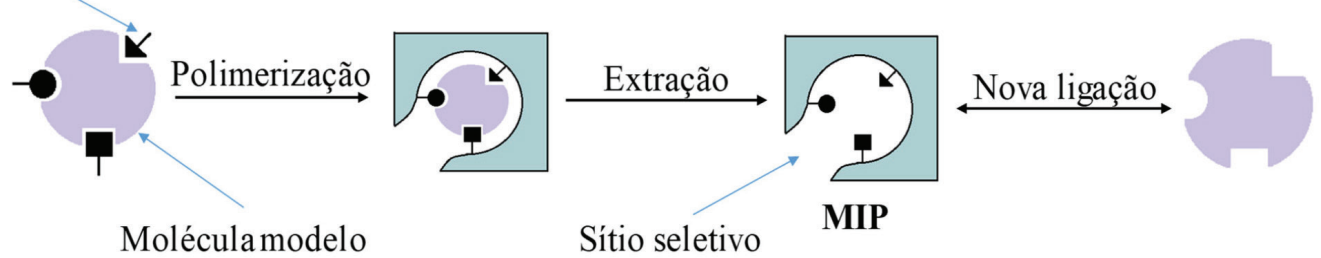

Figura 2. Esquema geral da síntese do MIP

matrizes complexas (e.g. sangue, urina e saliva), como a supressão iônica, sensibilidade baixa ao nível de traços e faixa dinâmica estreita. Estas limitações tem sido a força motriz para o desenvolvimento de novas abordagens que integrem de forma eficiente etapas de clean up, extração/enriquecimento do analito e ionização. Neste trabalho, apresentamos uma abordagem para melhorar a eficiência do PSI, frente a análise de matrizes complexas, que consiste em recobrir o papel, substrato do PSI, com um polímero molecularmente impresso (MIP - do inglês, molecularly imprinted polymer).

Os MIPs são materiais sintéticos que possuem sítios de reconhecimento capazes de religar um composto-alvo especificamente em detrimento de outros compostos estreitamente relacionados. Esses materiais são sintetizados via polimerização de monômeros funcionais e de reticulação em torno de uma molécula modelo, gerando um polímero em rede tridimensional altamente reticulado. ${ }^{24}$ Os monômeros são escolhidos de acordo com as suas capacidades de interagir com os grupos funcionais da molécula modelo. Quando ocorre a polimerização, a molécula modelo é extraída, gerando os sítios de ligação com a forma, tamanho e funcionalidade complementar do composto-alvo (Figura 2). Os MIPs são materiais estáveis, robustos e resistentes a uma ampla gama de $\mathrm{pH}$, solventes e temperatura, além de suas sínteses serem relativamente baratas e de fácil realização. ${ }^{25}$

O presente trabalho trata da combinação do MIP com o PSI-MS com o objetivo de se obter um método de análise seletivo (característica do MIP) e que forneça respostas analíticas em um curto espaço de tempo (característica do PSI-MS) para a detecção e quantificação de designer drugs em fluído biológico. Como designer drugs, foram utilizadas MDMA e MDA, e como fluído biológico, foi utilizado saliva sintética.

\section{PARTE EXPERIMENTAL}

\section{Preparo da saliva sintética}

A saliva sintética foi preparada de acordo com o protocolo proposto por Alshali et al. ${ }^{26}$ Foram dissolvidas $0,25 \mathrm{~g}$ de hidroxietil celulose em $400 \mathrm{~mL}$ de água ultrapurificada e a solução resultante foi submetida à agitação por $24 \mathrm{~h}$. Logo depois, foram adicionados: cloreto de potássio $\left(0,625 \mathrm{~g} \mathrm{~L}^{-1}\right)$, cloreto de cálcio dihidratado $\left(0,166 \mathrm{~g} \mathrm{~L}^{-1}\right)$, fosfato de potássio monobásico $\left(0,326 \mathrm{~g} \mathrm{~L}^{-1}\right)$, cloreto de magnésio hexahidratado $\left(0,059 \mathrm{~g} \mathrm{~L}^{-1}\right)$ e albumina sérica bovina $\left(0,00235 \mathrm{~g} \mathrm{~L}^{-1}\right)$. Após dissolver todos os reagentes, o $\mathrm{pH}$ foi ajustado para 6,75 utilizando hidróxido de potássio e o volume foi completado para $500 \mathrm{~mL}$.

\section{Síntese da membrana MIP}

A síntese da membrana MIP foi realizada a partir de uma adaptação da metodologia descrita por Sergeyeva et al. ${ }^{27}$ As membranas de celulose foram lavadas em um Soxlet com $400 \mathrm{~mL}$ de metanol por $4 \mathrm{~h}$ e secas à temperatura ambiente. Em seguida, as membranas foram submersas em uma solução de $150 \mathrm{mmol} \mathrm{L}^{-1}$ de benzofenona em acetona por 5 min e secas à temperatura ambiente. Foram, então, submersas em uma solução aquosa contendo $50 \mathrm{mmol} \mathrm{L}^{-1}$ de ácido metacrílico, $150 \mathrm{mmol} \mathrm{L}^{-1}$ de etileno dimetacrilato, $10 \mathrm{mmol} \mathrm{L}^{-1} \mathrm{de}$ MDA ou MDMA. Posteriormente, foram expostas à radiação UV (ultravioleta) por 15 min utilizando um comprimento de onda de $254 \mathrm{~nm}$. Por fim, as membranas foram lavadas novamente no sistema de Soxlet com $400 \mathrm{~mL}$ de metanol por $4 \mathrm{~h}$ para retirada do molde. Após secas, as membranas foram pesadas com o intuito de avaliar a eficiência de remoção do molde. Seguindo a mesma metodologia, foi sintetizado um polímero não-impresso (NIP), porém, não contendo a molécula modelo, para agir como um controle (através da comparação entre MIP e NIP) na verificação da seletividade do MIP para as moléculas desejadas. As membranas (MIP e NIP) foram cortadas em formato triangular equilátero com altura de $1 \mathrm{~cm}$.

\section{MIP-PSI-MS}

As membranas poliméricas foram imersas por $5 \mathrm{~min}$ em $1 \mathrm{~mL}$ de soluções de diferentes concentrações de MDA ou MDMA (amostras fornecidas pela Polícia Civil do Estado do Espírito Santo) em saliva sintética. Em seguida, foram lavadas com água deionizada para remoção da saliva e secadas por 15 min à temperatura ambiente. Para a análise de PSI-MS (Figura 3), a membrana polimérica foi presa por sua base em um grampo metálico onde uma alta voltagem foi aplicada. Os parâmetros experimentais foram baseados em trabalhos anteriores desenvolvidos no nosso grupo utilizando PSI-MS..$^{810-12}$ A membrana foi posicionada a uma distância de aproximadamente

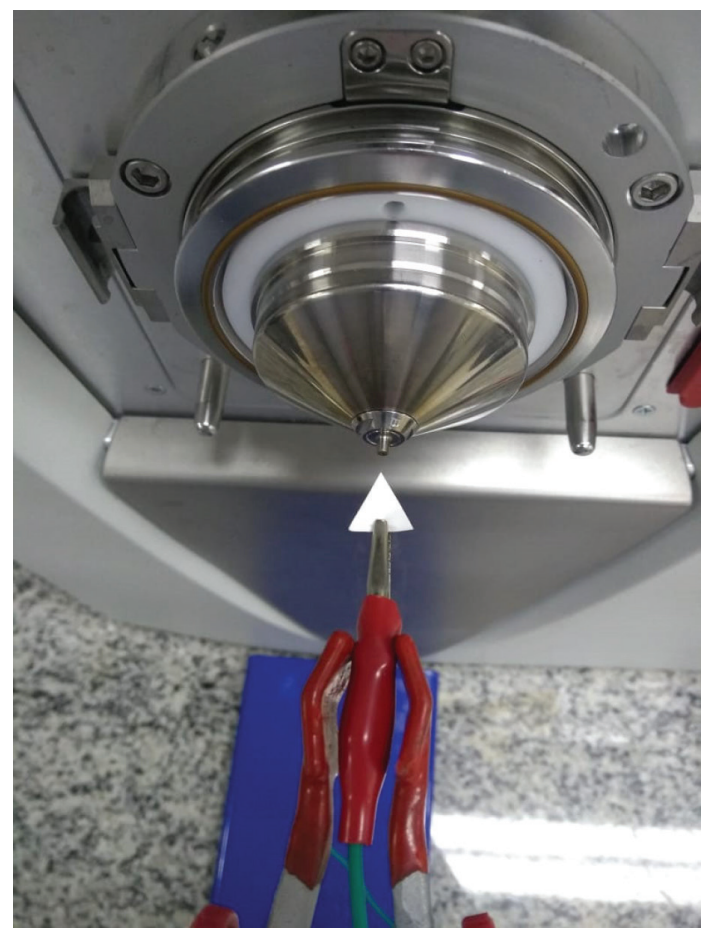

Figura 3. Sistema MIP-PSI homemade acoplado ao espectrômetro de massas LCQ Fleet 
$4 \mathrm{~mm}$ da entrada do espectrômetro de massas. Utilizaram-se $10 \mu \mathrm{L}$ de metanol com $0,1 \%$ de ácido fórmico para análises no modo positivo. Para obtenção dos espectros de massas, foi utilizado um espectrômetro de massas Thermo Scientific Ion trap LCQ Fleet. Foram utilizados os seguintes parâmetros instrumentais: voltagem do spray: $3,5 \mathrm{kV}$; temperatura do capilar: $275^{\circ} \mathrm{C}$; tensão do capilar: $35 \mathrm{~V}$; tube lens: $50 \mathrm{~V}$; energia de colisão: $23 \mathrm{eV}$.

\section{Validação analítica}

O método MIP-PSI-MS foi validado parcialmente de acordo com a RDC n 166 de 24 de julho de 2017 da Agência Nacional de Vigilância Sanitária (Anvisa) ${ }^{28}$ que tem por objetivo principal regular e controlar a área sanitária de serviços e produtos, incluindo a validação para métodos analíticos. Os critérios analisados foram: linearidade, limites de detecção (LD) e quantificação (LQ), precisão, exatidão, recuperação e sensibilidade.

A linearidade é a capacidade do método em fornecer resultados proporcionais à concentração do analíto em um intervalo determinado. ${ }^{29} \mathrm{~A}$ forma comum de identificar a linearidade é analisar o coeficiente de correlação (R) das curvas analíticas e, de acordo com a Anvisa, o valor do R deve ser maior que 0,990. Neste trabalho, a curva analítica foi construída monitorando o aumento da intensidade do fragmento mais intenso do MDMA e MDA, o $\mathrm{m} / \mathrm{z} 163$ para ambos. $\mathrm{O}$ íon $\mathrm{m} / z, 163$ é resultante de um experimento de $\mathrm{MS}^{2}$ nas moléculas de interesse. Para a construção das curvas, o MDMA e MDA foram dissolvidos em saliva sintética para obter as seguintes concentrações: $25,90,180,270,360,410$ e $500 \mu \mathrm{g} \mathrm{L}^{-1}$.

O limite de detecção (LD) é a menor quantidade detectável porém não quantificável do analito. Já o limite de quantificação (LQ) é a menor quantidade de analito quantificável de forma precisa e exata. ${ }^{30}$ O LD e o LQ foram determinados baseados em 3 e 10 vezes a razão sinal-ruído, respectivamente.

As curvas de correlação para avaliar a capacidade do método em quantificar analitos em concentrações próximas a valores reais foram construídas utilizando as seguintes concentrações: 45, 90, 140, 180, 230, 270, 320, 360, 380, 410, 450, $500 \mu \mathrm{g} \mathrm{L}^{-1}$.

A exatidão é o quão próximo o valor dos resultados obtidos pelo método se aproxima dos valores verdadeiros. A precisão é a proximidade dos resultados em várias medidas de uma mesma amostra. ${ }^{30} \mathrm{~A}$ exatidão e precisão foram realizadas intra $(n=5)$ e inter-dia $(n=3)$ utilizando as seguintes concentrações: 90, 270 e $500 \mu \mathrm{g} \mathrm{L}^{-1}$, respectivas concentrações baixa, média e alta da curva de calibração. A exatidão foi obtida pela seguinte equação:

$$
\text { Exatidão }=\frac{X i-X v}{X v} 100
$$

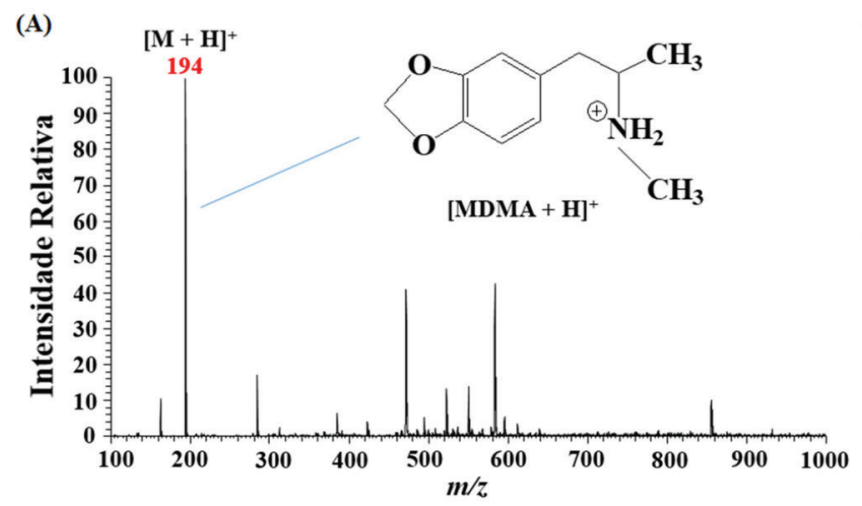

em que Xi é a concentração analisada e Xv é a concentração nominal. A precisão foi determinada através da seguinte equação:

$$
\text { Precisão }=\left(\frac{\mathrm{S}}{\mathrm{Xi}}\right) 100
$$

sendo S o desvio padrão.

A recuperação está diretamente ligada à exatidão, pois mostra a quantidade de analito recuperada na análise de acordo com a quantidade de analito real na amostra. ${ }^{29} \mathrm{~A}$ recuperação foi realizada com as mesmas concentrações utilizadas nos testes de exatidão e precisão, e foi calculada através da seguinte equação:

$$
\text { Recuperação }=\frac{X i-X o}{X v} 100
$$

em que Xo é concentração original do analito na amostra.

\section{RESULTADOS E DISCUSSÃO}

\section{Análise de MDMA $e$ MDA em saliva}

A geometria triangular do papel foi largamente exposta na literatura. ${ }^{13,14}$ Geometrias distintas no papel provocam variabilidade na intensidade do campo elétrico e na corrente aplicada. ${ }^{14} \mathrm{~A}$ forma geométrica mais adequada é a triangular.

A Figura 4 ilustra os espectros de massas obtidos por MIP-PSI-MS da saliva artificial dopada com $500 \mu \mathrm{g} \mathrm{L}^{-1}$ de $\operatorname{MDMA}(\mathrm{m} / \mathrm{z}, 194)$ e MDA $(\mathrm{m} / \mathrm{z}$ 180). É possível notar a alta intensidade dos íons protonados de ambos analitos. A ausência de íons intensos oriundos da saliva, demonstra a eficácia do MIP-PSI-MS em evitar o efeito de supressão iônica comum em amostras complexas.

A Figura 5 ilustra o espectro de massas obtido por MIP-PSI-MS da saliva sintética, branco, utilizando a membrana MIP-MDMA (Figura 5a) e MIP-MDA (Figura 5b). Note que os íons $[\mathrm{M}+\mathrm{H}]^{+} \mathrm{de}$ $\mathrm{m} / \mathrm{z}, 194$ e $\mathrm{m} / \mathrm{z} 180$ referentes ao MDMA e MDA mostrados anteriormente (Figura 4) não foram detectados. Neste estudo, priorizou o uso da molécula alvo como molde. A Figura 5 mostra que processo de lavagem para retirada do molde foi eficiente. Andersson et al. ${ }^{31}$ propôs o uso de uma molécula análoga ao analito alvo como template, a fim de se evitar falsos positivos caso a etapa de lavagem não seja eficiente. Entretanto, a principal desvantagem do uso de moldes análogos é a capacidade de reconhecimento molecular menor quando comparadas aos que usam a molécula alvo como molde. ${ }^{32} \mathrm{O}$ uso do mesmo alvo como template, neste trabalho, não interferiu na análise do analito de interesse.

Figura 4. Espectro de massas obtido por MIP-PSI(+)-MS com $500 \mu \mathrm{g} L^{-1}$ de MDMA (A) e MDA (B) em saliva artificial

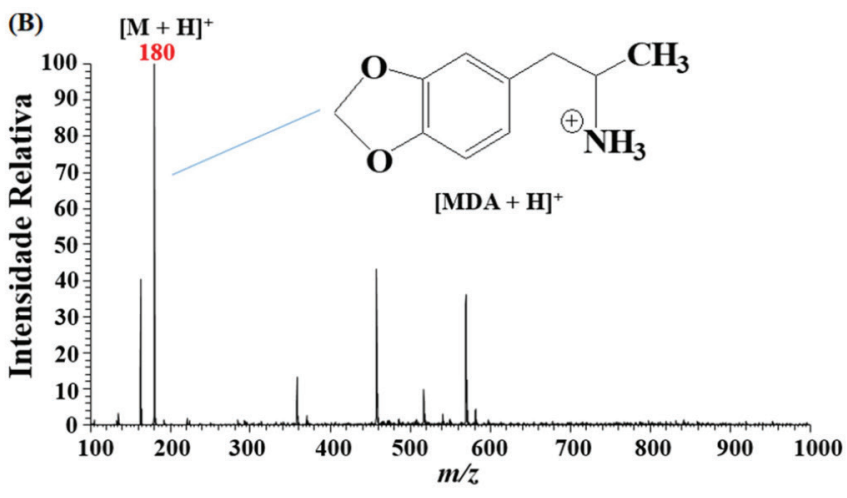




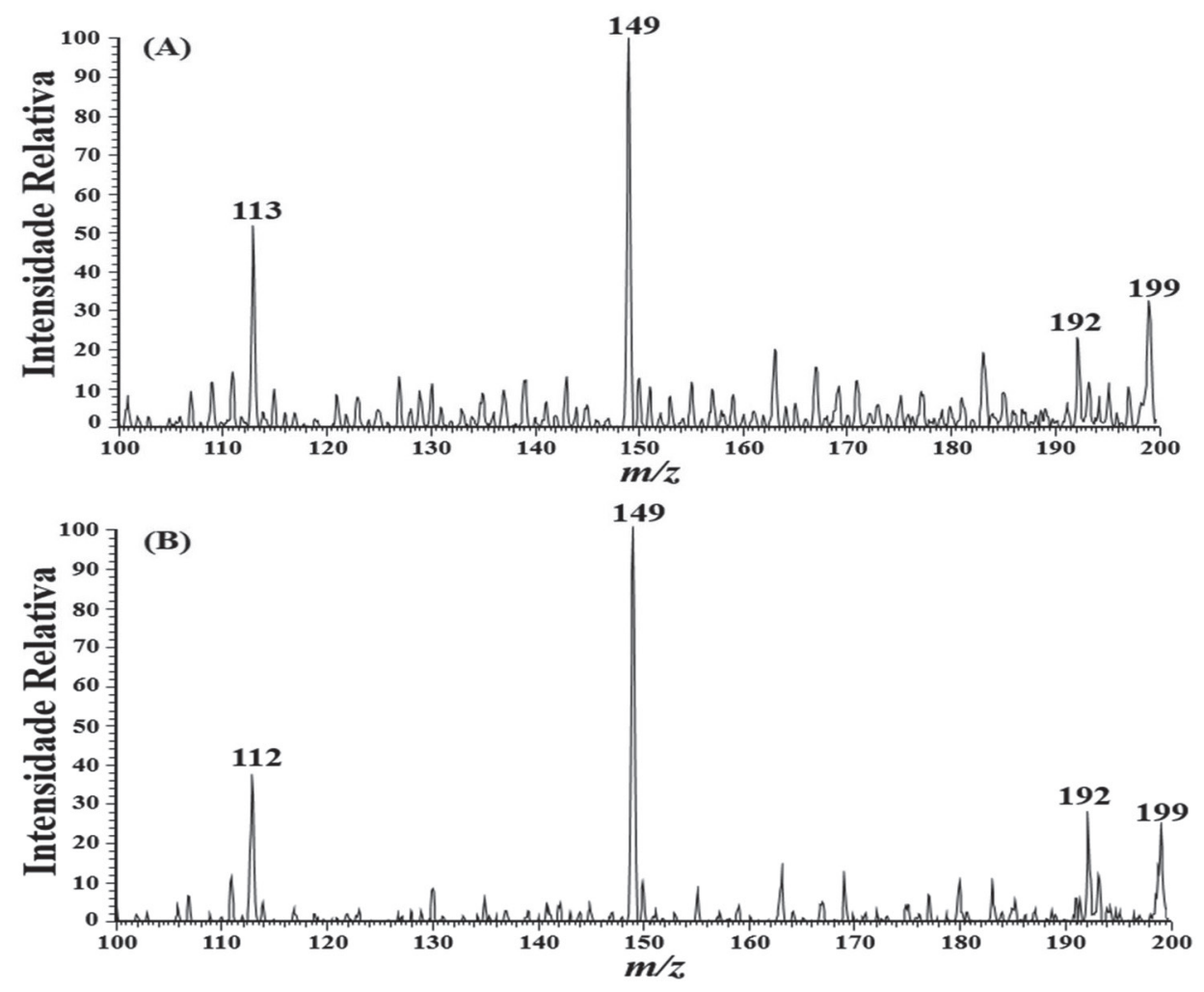

Figura 5. Espectro de massas obtido por MIP-PSI(+)-MS com molde de MDMA(A) e MDA (B) sem adição de MDMA e MDA na saliva sintética

\section{Teste de seletividade}

A capacidade de sequestrar os analitos alvos dos MIPs foi avaliada comparando as intensidades obtidas com as membranas MIP e NIP. A Figura 6a ilustra um gráfico de barras que demonstra as intensidades dos íons protonados $[\mathrm{M}+\mathrm{H}]^{+}$do MDMA $(\mathrm{m} / \mathrm{z}, 194), \mathrm{mCPP}(\mathrm{m} / \mathrm{z}, 197)$, DOB $(\mathrm{m} / z$ 275) e LSD $(\mathrm{m} / \mathrm{z}$ 324) utilizando as membranas MIP-MDMA e NIP. Ao verificar as análises de NIP e MIP com o mCPP, DOB e LSD, observa-se uma pequena diferença entre as intensidades dos sinais. Todavia, para as análises com o MDMA, a intensidade do sinal obtida por MIP-MDMA foi muito maior do que a obtida por NIP. Isso demonstra que a membrana MIP-MDMA possui uma alta seletividade para a molécula de MDMA, em detrimento das moléculas de mCPP, DOB e LSD. Esse resultado era esperado, uma vez que o MDMA foi utilizado como template para a síntese do MIP. A Figura $6 \mathrm{~b}$ demonstra a mesma abordagem, porém, utilizando a membrana MIP-MDA. A maior intensidade é encontrada na análise do composto MDA, demonstrando a seletividade da membrana para esse composto.
Os espectros de massas de dissociação do MDMA e MDA são mostrados na Figura 7. Note que na Figura 7(A), espectro de MIP-PSI-MS/MS do MDMA, a principal perda é evidenciada pela transição $m / z, 194 \rightarrow 163$, referente à perda de um grupo metilamina $\left(\mathrm{NH}_{2} \mathrm{CH}_{3}\right)$. Na Figura 7(B), a transição $m / z, 163 \rightarrow 135$, decorrente de um experimento de $\mathrm{MS}^{3}$, é referente à perda de etileno $\left(\mathrm{CH}_{2} \mathrm{CH}_{2}\right)$; a transição $\mathrm{m} / \mathrm{z}, 163 \rightarrow 133$ é referente à perda de formaldeído $\left(\mathrm{CH}_{2} \mathrm{O}\right)$, devido a um rearranjo no grupo metilenodioxi; e a transição $\mathrm{m} / \mathrm{z}, 135 \rightarrow 105$ é devido à outra perda de um grupo formaldeído $\left(\mathrm{CH}_{2} \mathrm{O}\right)$. Na Figura 7(C) é mostrado o espectro de PSI-MS/MS do MDA. A perda mais importante é evidenciada pela transição $m / z, 180 \rightarrow 163$, referente à perda de amônia $\left(\mathrm{NH}_{3}\right)$, comum em aminas primárias. Na Figura 7(D), a transição $m / z, 163 \rightarrow 135$, decorrente de um experimento de $\mathrm{MS}^{3}$, é referente à perda de etileno $\left(\mathrm{CH}_{2} \mathrm{CH}_{2}\right)$; a transição $\mathrm{m} / \mathrm{z} 163 \rightarrow 133$ é devido à perda de formaldeído $\left(\mathrm{CH}_{2} \mathrm{O}\right)$, em virtude de um rearranjo no grupo metilenodioxi; e a transição $\mathrm{m} / z, 135 \rightarrow 105$ também é referente à outra perda de formaldeído $\left(\mathrm{CH}_{2} \mathrm{O}\right)$. Essas perdas também são mostradas por Schepens et al., ${ }^{33}$

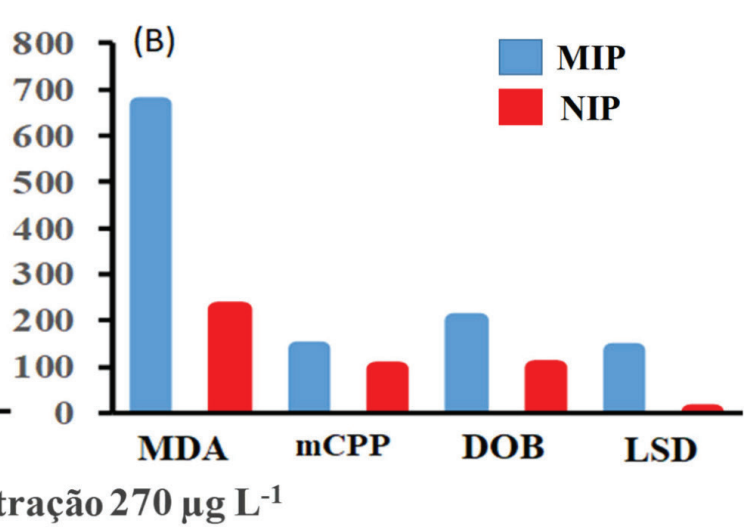

Figura 6. Comparativo das intensidades absolutas do íon $[\mathrm{M}+\mathrm{H}]^{+}$do MDMA e MDA utilizando o MIP e NIP-PSI-MS 
que fizeram uma rápida quantificação de drogas de abuso em fluido oral utilizando a MS.

\section{Validação analítica}

Para verificar a performance analítica da técnica, curvas analíticas (Figura 8) foram construídas com diferentes concentrações de MDMA e MDA em saliva sintética através do monitoramento das transições $m / z, 194 \rightarrow 163$ (MDMA) e $m / z, 180 \rightarrow 163$ (MDA). O método MIP-PSI-MS apresentou linearidades com o R > 0,99 para ambas as designer drugs, em conformidade com as diretrizes da Anvisa. Os valores de LD e LQ obtidos para o MDMA foram de $1,11 \mu \mathrm{g} \mathrm{L}^{-1}$ e $18,73 \mu \mathrm{g} \mathrm{L}{ }^{-1}$, respectivamente. Já para o MDA, o LD e LQ foram 3,42 $\mu \mathrm{g} \mathrm{L}^{-1}$ e 21,66 $\mu \mathrm{g} \mathrm{L}^{-1}$, respectivamente. Schepens et al. ${ }^{33}$ mostraram valores próximos de LD e LQ para o MDMA (ambos $\left.<5 \mu \mathrm{g} \mathrm{L}^{-1}\right)$ e para o MDA $\left(10 \mu \mathrm{g} \mathrm{L} \mathrm{L}^{-1}\right.$ e $\left.25 \mu \mathrm{g} \mathrm{L}{ }^{-1}\right)$ com o método de Cromatografia Liquida acoplada à MS (LC-MS) para rápida confirmação e quantificação de drogas de abuso em saliva sintética. Ishikawa et al. ${ }^{34}$ também mostraram valores próximos de LD e LQ para o MDA, 2,5 $\mu \mathrm{g} \mathrm{L}^{-1}$ e $10 \mu \mathrm{g} \mathrm{L}^{-1}$, e para o MDMA, $1 \mu \mathrm{g} \mathrm{L}^{-1}$ e $10 \mu \mathrm{g} \mathrm{L}^{-1}$, em fluídos biológicos utilizando Cromatografia Gasosa acoplada à Espectrometria de Massa (CG-MS). Portanto, o método de MIP-PSI-MS atingiu resultados próximos aos de técnicas clássicas utilizadas para análises de drogas.

A Figura 9 demonstra curvas analíticas que correlacionam concentrações reais (quantidades das drogas concentradas na saliva) com concentrações obtidas por MIP-PSI-MS para as drogas MDA e MDMA. Esse teste demonstra a capacidade da técnica em quantificar compostos em valores próximos aos reais. De acordo com a Figura 9a (MDMA) e Figura 9b (MDA), é possível notar correlações entre as concentrações reais e medidas para ambas as drogas, apresentando valores de $\mathrm{R}$ maiores que 0,99 . Desse modo, o método MIP-PSI-MS apresenta um alto grau de confiabilidade para determinação das drogas MDMA e MDA em concentrações próximas do valor existente na amostra, com um potencial uso para quantificação de designer drugs em amostras reais.
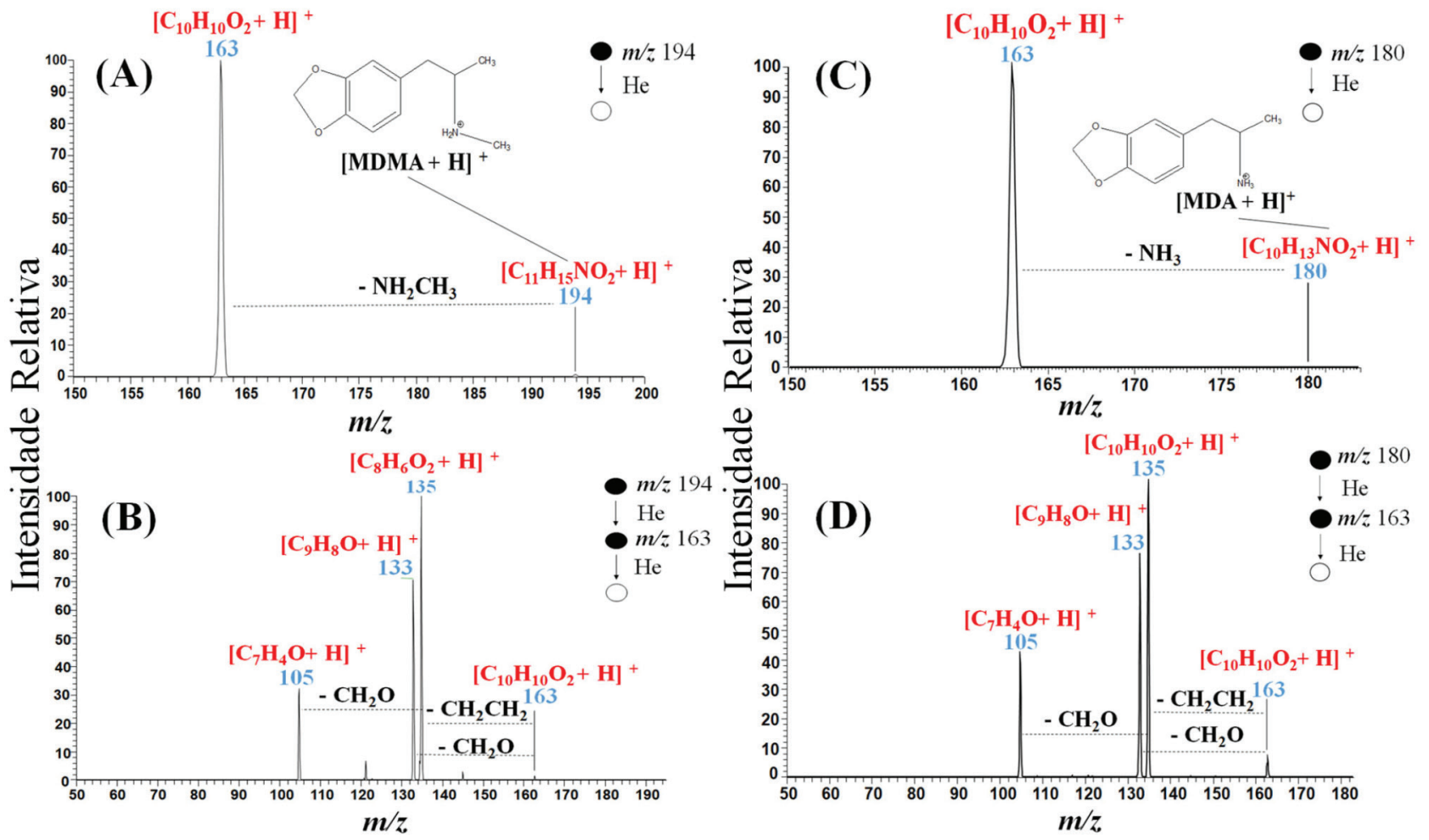

Figura 7. Espectro de MIP-PSI(+)-MS/MS do MDMA (A) e (B) e MDA (C) e (D) em saliva sintética
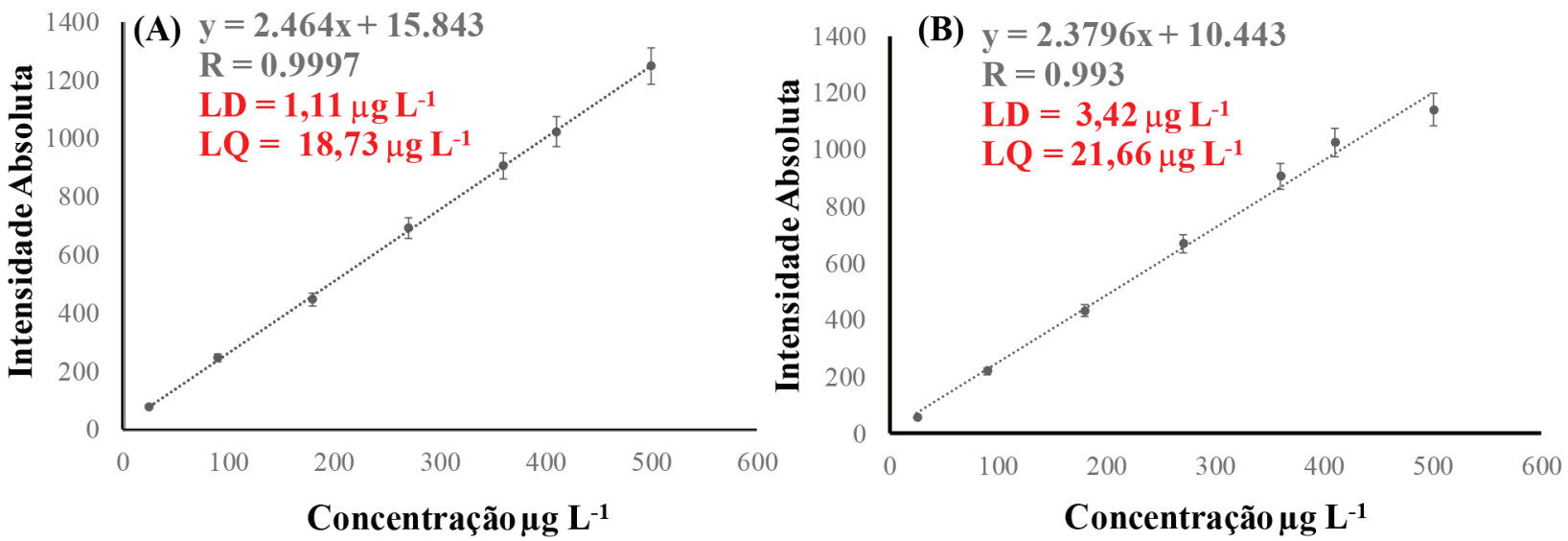

Figura 8. Curva analítica, linearidade (R), LD e LQ obtidos por MIP-PSI(+)-MS para MDMA (A) e MDA (B) 

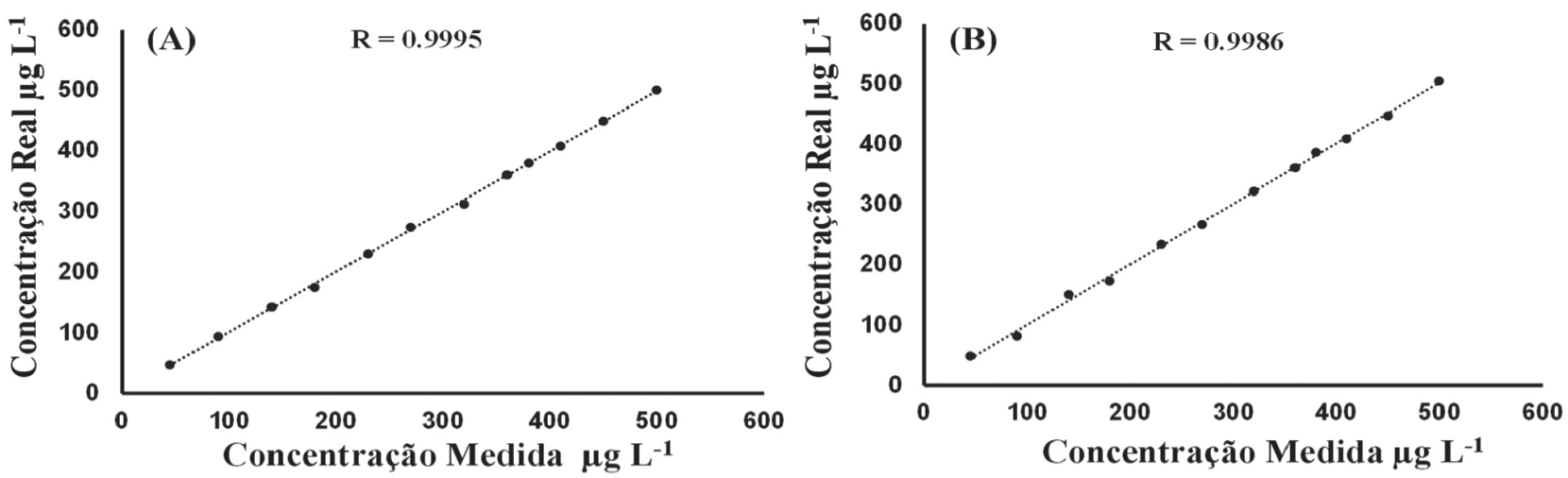

Figura 9. Curva analítica que demonstra a correlação entre concentrações reais e medidas em saliva utilizando MIP-PSI(+)-MS para o MDMA (A) e MDA (B)

A Tabela 1 retrata os resultados de precisão, exatidão e recuperação do MIP-PSI-MS para o MDMA. Os valores de precisão intra-dia variaram entre 0,66 e 1,52\%, e inter-dia entre 0,86 e 1,35\%. Já os valores de exatidão intra-dia variaram de 0,51 a $3,92 \%$, e inter-dia de 0,84 a $3,84 \%$. A recuperação esteve próxima de $100 \%$.

Tabela 1. Precisão, exatidão e recuperação para o MDMA por MIP-PSI-MS

\begin{tabular}{|c|c|c|c|c|}
\hline \multicolumn{2}{|c|}{ Concentração nominal $\left(\mu \mathrm{g} \mathrm{L}^{-1}\right)$} & 90 & 270 & 500 \\
\hline \multicolumn{2}{|c|}{ Concentração obtida $\left(\mu \mathrm{g} \mathrm{L}^{-1}\right)$} & 91,73 & 271,05 & 502,33 \\
\hline \multirow[t]{2}{*}{ Intra-dia $(\%)$} & Precisão & 1,52 & 0,66 & 0,84 \\
\hline & Exatidão & 3,92 & 1,82 & 0,51 \\
\hline \multirow[t]{2}{*}{ Inter-dia (\%) } & Precisão & 1,14 & 0,86 & 1,35 \\
\hline & Exatidão & 3,84 & 1,39 & 0,84 \\
\hline \multicolumn{2}{|c|}{ Recuperação (\%) } & 103,92 & 101,81 & 100,54 \\
\hline
\end{tabular}

Os valores de precisão, exatidão e recuperação para o MDA estão dispostos na Tabela 2. Os resultados de precisão intra-dia variaram de 0,49 a $1,53 \%$, e inter-dia de 0,96 a $2,26 \%$. Os valores de exatidão intra-dia variaram entre 0,35 e $1,98 \%$, e inter-dia entre 0,35 e $1,98 \%$. O método apresentou valores de recuperação próximos de 100\%. De acordo com a RDC no 166 de 24 de julho de 2017 da ANVISA, ${ }^{15}$ valores de precisão e exatidão até $20 \%$ são aceitos para amostras biológicas. Os valores de recuperação próximos de $100 \%$, como mostrado aqui, evidencia uma maior eficiência do método MIP-PSI-MS perante ao método de determinação de drogas de abuso em biofilme dental, utilizando LC-MS/MS, descrito por Henkel et al. ${ }^{35} \mathrm{O}$ parâmetro da recuperação encontra-se dentro do intervalo estimado pela Anvisa, de 80-120\%, que é o indicado desde que o método seja preciso e exato.

Tabela 2. Precisão, exatidão e recuperação para o MDA por MIP-PSI-MS

\begin{tabular}{|c|c|c|c|c|}
\hline \multicolumn{2}{|c|}{ Concentração nominal $\left(\mu \mathrm{g} \mathrm{L}^{-1}\right)$} & 90 & 270 & 500 \\
\hline \multicolumn{2}{|c|}{ Concentração obtida $\left(\mu \mathrm{g} \mathrm{L}^{-1}\right)$} & 90,66 & 273,68 & 503,17 \\
\hline \multirow[t]{2}{*}{ Intra-dia $(\%)$} & Precisão & 1,53 & 0,87 & 0,49 \\
\hline & Exatidão & 0,64 & 1,98 & 0,51 \\
\hline \multirow[t]{2}{*}{ Inter-dia (\%) } & Precisão & 2,26 & 1,05 & 0,96 \\
\hline & Exatidão & 0,64 & 1,98 & 0,35 \\
\hline \multicolumn{2}{|c|}{ Recuperação (\%) } & 100,64 & 101,98 & 100,63 \\
\hline
\end{tabular}

\section{CONCLUSÃO}

Neste trabalho foram demonstradas análises de designer drugs em saliva utilizando MIP-PSI-MS, um método que combina a síntese do
MIP sobre uma membrana de celulose que é utilizada como substrato em análises de PSI-MS. O método proposto apresenta a vantagem de fornecer resultados de forma rápida, uma vez que o MIP pode ser produzido em aproximadamente $4 \mathrm{~h}$ e a análise por PSI-MS pode ser realizada em segundos. Além disso, o MIP-PSI-MS não requer etapas laboriosas de preparo de amostras para fornecer resultados analíticos adequados. Estas características fazem do MIP-PSI-MS um método com potencial aplicação em laboratórios de perícia, devido à necessidade de detecção e quantificação de designer drugs de forma rápida e precisa. A performance analítica do MIP-PSI-MS foi avaliada através da análise das drogas MDMA e MDA em saliva sintética. O MIP-PSI-MS apresentou uma elevada seletividade para o MDMA e MDA, apresentando íons mais intensos para ambas as drogas em relação a analitos com estruturas moleculares diferentes. A linearidade das curvas analíticas foram determinadas com $R>0,99$. Valores de LD e LQ obtidos para o MDMA foram de $1,11 \mu \mathrm{g} \mathrm{L} \mathrm{L}^{-1} \mathrm{e} 18,73 \mu \mathrm{g} \mathrm{L}^{-1}$, respectivamente, e para o MDA, 3,42 $\mu \mathrm{g} \mathrm{L}^{-1}$ e 21,66 $\mu \mathrm{g} \mathrm{L}^{-1}$, respectivamente, em equivalência a outros métodos de análises de drogas clássicas já existentes. Os valores de precisão e exatidão foram determinados abaixo de $4 \%$, e as recuperações próximas de $100 \%$. Estes resultados se encontram dentro dos parâmetros de validação de métodos analíticos descritos pela RDC $\mathrm{n}^{\circ} 166$ de 24 de julho de 2017 da Anvisa.

\section{AGRADECIMENTOS}

Ao CNPq, à CAPES e à FAPEG, pelo apoio e suporte financeiro.

\section{REFERÊNCIAS}

1. Bulcão, R.; Garcia, S. C.; Limberger, R. P.; Baierle, M.; Arbo, M. D.; Chasin, A. A. M.; Thiesen, F. V.; Tavares, R.; Quim. Nova 2012, 35, 149.

2. http://www.unodc.org/documents/scientific/NPS_2013_SMART.pdf, acessada em junho de 2019.

3. Henderson, G. L.; J. Forensic. Sci. 1988, 33, 569.

4. Costa, J. L.; Matta, A. A.; J. Chromatogr. B 2004, 811, 41.

5. Alberici, R. M.; Simas, R. C.; Sanvido, G. B.; Romão, W.; Lalii, P. M.; Benassi, M.; Cunha, I. B. S.; Eberlin, M. N.; Anal. Bioanal. Chem. 2010, 398, 265.

6. Mendes, P. P. T.; Pereira, I.; Ferreira, R. M.; Chaves, R. A.; Vaz, G. B.; Anal. Methods 2017, 9, 1.

7. Correa, N. D.; Santos, M. J.; Eberlin, S. L.; Eberlin, N. M.; Teunissen, F. S.; Anal. Chem. 2016, 88, 2515.

8. Pereira, I.; Carvalho, T. C. de; Romão, W.; Filgueiras, P. R.; Laviola, B. G.; Rodrigues, C. M.; Abdelnur, P. V.; Vaz, B. G.; J. Braz. Chem. Soc. 2017, 28, 1461

9. Wang, H.; Liu, J.; Cooks, R. G.; Ouyang, Z.; Angew. Chem. 2010, 49, 877. 
10. Pereira, I.; Rodrigues, S. R. M.; Carvalho, T. C. de; Carvalho, V. V.; Lobón, G. S.; Bassane, J. F. P.; Domingos, E.; Romão, W.; Augusti, R.; Vaz, B. G.; Anal. Methods 2016, 8, 6023.

11. Pereira, I.; Rodrigues, M. F.; Chaves, A. R.; Vaz, B. G.; Talanta 2018, 178, 507.

12. Domingos, E.; Carvalho, T. C. de; Pereira, I.; Vasconcelos, G. A.; Thompson, C. J.; Augusti, R.; Rodrigues, R. R. T.; Tose, L. V.; Santos, H.; Araujo, J. R.; Vaz, B. G.; Romão, W.; Anal. Methods 2017, 9, 4400.

13. Liu, J.; Wang, H.; Manicke, N. E.; Lin, J. M.; R.; Cooks, G. R.; Ouyang, Z.; Anal. Chem. 2010, 82, 2463.

14. Yang, Q.; Manicke, N.E.; Wang, H.; Petucci, C.; Cooks, R.G.; Ouyang, Z.; Anal Bioanal. Chem. 2012, 404, 1389.

15. Manicke, N. E.; Neil, P.A.-R.; Ouyang, S. Z.; Cooks, R. G.; J. Am. Soc. Mass Spectrom. 2011, 22, 1501.

16. Espy, R. D.; Manicke, N. E.; Ouyang, Z.; Cooks, R. G.; Analyst 2012, 137, 2344

17. Yang, Q.; Wang, H.; Maas, J. D.; Chappell, W. J.; Manicke, N. E.; Cooks, R. G.; Ouyang, Z.; Int. J. Mass Spectrom. 2012, 312, 201.

18. Cooks, R. G.; Manicke, N. E.; Dill, A. L.; Ifa, D. R.; Eberlin, L. S.; Costa, A. B.; Wang, H.; Huang, G.; Ouyang, Z.; Faraday Discuss. 2011, 149, 247.

19. Shi, R.-Z.; Gierari, E. T. M. E.; Manicke, N. E.; Faix, J. D.; Clin. Chim. Acta 2015, 441, 99.

20. Yannell, K. E.; Kesely K. R.; Chien H. D.; Kissinger C. B.; Cooks R. G.; Anal. Bioanal. Chem. 2017, 409, 121.

21. Kennedy, J. H.; Palaty, J.; Gill, C. G.; Wiseman, J. M.; Rapid Commun. Mass Spectrom. 2018, 32, 1280.
22. Tavares, L. S.; Carvalho, T. C.; Romão, W.; Vaz, B. G.; Chaves, A. R.; J. Am. Soc. Mass Spectrom. 2018, 29, 566.

23. Jhang, C. S.; Lee, H.; He, Y. S.; Liu, J. T.; Lin, C. H.; Electrophoresis 2012, 33, 3073.

24. Cormack, P. A. G.; Elorza, A. Z.; J. Chromatogr. B 2004, 804, 173.

25. Martín-Esteban, A.; TrAC --Trends Anal. Chem. 2013, 45, 169.

26. Alshali, R. Z.; Salim, N. A.; Satterthwaite, J. D.; Silikas, N.; J. Dent. 2015, $2528,1$.

27. Sergeyeva, T. A.; Matuschewski, H.; Piletsky, S. A.; Bendig, J.; Schedler, U.; Ulbricht, M.; J. Chromatogr. A 2001, 907, 89.

28. http://portal.anvisa.gov.br/documents/10181/2721567/RDC_166_201_ COMP.pdf/d5fb92b3-6c6b-4130-8670-4e3263763401, acessada em junho de 2019.

29. Brito, N. M.; Junior, O. P. A.; Polese, L.; Ribeiro, M. L.; Pesticidas 2003, 13, 129.

30. Ribani, M.; Bottoli, C. B. G.; Collins C. H.; Jardim, I. C. F. S.; Quim. Nova 2004, 27, 771

31. Andersson, L. I.; Paprica, A.; Arvidsson, T.; Chromatographia 1997, 46, 57.

32. Esteban, M. A.; Trends Environ. Anal. Chem. 2016, 9, 8.

33. Schepens, E.; Inman, S.; McCullough, B. J.; Hopley, C.; Forensic Chem. 2017, 4, 75 .

34. Ishikawa, A. A.; Bordin, D. M.; de Campos, E. G.; Blanes, L.; Doble, P.; Martinis, B. S. D.; J. Anal. Toxicol. 2018, 42, 661.

35. Henkel, K.; Altenburger, M. J.; Auwärter, V.; Neukamm, M. A.; Talanta 2018, 176, 360 . 